\title{
Multiple Transversion Abnormalities
}

National Cancer Institute

\section{Source}

National Cancer Institute. Multiple Transversion Abnormalities. NCI Thesaurus. Code C45657.

Multiple point mutations in a DNA sequence from eukaryotic or prokaryotic org anisms in which each mutation occurs by substitution of a purine base for a pyrimidine base or vice versa. These abnormalities can be either heritable or occur somatically. 